\title{
The effects of Hebb repetition learning and temporal grouping in immediate serial recall of spatial location
}

\author{
Momoe Sukegawa $^{1} \cdot$ Yoshiyuki Ueda $^{2} \cdot$ Satoru Saito $^{3}$ \\ Published online: 22 March 2019 \\ (C) The Psychonomic Society, Inc. 2019
}

\begin{abstract}
The Hebb repetition effect is a phenomenon in which a repeated presentation of the same list increases the performance in immediate serial recall. This provided the theoretical basis for a core assumption of the Atkinson and Shiffrin model regarding information transfer from short-term memory to long-term memory. The Hebb repetition effect was originally reported for the verbal domain, but subsequent studies found similar phenomena using visuospatial paradigms, for example, in serial-order memory for dot locations. The present study examined in two experiments the effects of presentation timing of nine spatial locations on Hebb repetition learning. In Experiment 1, the Hebb repetition effects were observed for spatial locations with constant timing presentation as well as temporal grouping presentation. In the latter condition, all lists were presented with the same temporal structure, that is, temporal pauses were inserted after the third and sixth serial positions. This manipulation led to a better recall performance in comparison with the constant presentation, but did not interact with the repetition. In Experiment 2, the Hebb list was presented with a different temporal structure in every repetition in the random-grouping condition. Although this manipulation is known to eliminate or weaken the Hebb effect in the verbal domain, we observed stable repetition effects in this experiment. This suggests that there might be some domain-specific mechanisms in Hebb repetition learning. These results may facilitate the development of theories of the relationship between short-term and long-term memory.
\end{abstract}

Keywords Hebb repetition learning · Temporal grouping · Visuospatial short-term memory

\section{Introduction}

Serial-order information is ubiquitous in our cognitive activities. It is a fundamental requirement to process such information for the functions of most mental processes (e.g., Lashley, 1951). This is true even for cognitive processes operating over a short period of time: Word order must be retained and processed during a short conversation and phoneme order within a word must be retained just before the production of a word. In those cases, the temporary retention of serial-order information is essential for immediate actions. At the same time, the immediate use of serial-order information is supported by long-term knowledge, for example, vocabulary knowledge to process

Satoru Saito

saito.satoru.2z@kyoto-u.ac.jp

Faculty of Education, Kyoto University, Kyoto, Japan

Kokoro Research Center, Kyoto University, Kyoto, Japan

Department of Cognitive Psychology in Education, Graduate School of Education, Kyoto University, Yoshida-Honmachi, Sakyo-ku, Kyoto 606-8501, Japan word information. Therefore, mechanisms for the retention of serial-order information over a short term and their interactions with long-term serial-order knowledge have been one of the most important research topics in psychological science (e.g., Hurlstone, Hitch, \& Baddeley, 2014; Thorn \& Page, 2009).

Research endeavors have been devoted to the examination of this relationship between short-term retention of serial order and long-term sequential knowledge through a specific research paradigm: the Hebb repetition paradigm (Hebb, 1961). The original experiment by Donald Hebb (1961) required participants to perform immediate serial recall of 24 sequences (i.e., 24 trials) of nine digits while presenting the same sequence in every third trial. The repeated sequences are called the Hebb lists, and those sequences are known to show the gradual improvement of memory performance over repetitions compared to non-repeated sequences or filler lists. Note that this Hebb repetition effect seems to occur regardless of the participant's awareness of the repetition, indicating that Hebb repetition learning is at least partly implicit (e.g., McKelvie, 1987). Another notable characteristic of the Hebb repetition effect is the incidental nature of its long-term learning as pointed out in the influential paper by Atkinson and Shiffrin (1968, p. 103). That is, although participants did not intend to learn the given 
sequences for longer-term use, they certainly gradually developed the ability to recall the Hebb sequences over repetition, indicating the presence of long-term learning. This fact led Atkinson and Shiffrin to one of the core assumptions in their model of human memory: "...throughout the period that information resides in the short-term store, transfer takes place to long-term store" (p. 103). Some of the later studies, however, have questioned the assumption that short-term retention of information is necessary for long-term learning (e.g., Shallice \& Warrington, 1970). Nevertheless, it is argued that information transfer from short-term memory (STM) to long-term memory (LTM) can operate, but in a domain-specific manner (e.g., Baddeley, Gathercole, \& Papagno, 1998) although this has been confirmed mainly in the verbal domain.

The precise mechanisms of the Hebb repetition effect are still to be explored, and there are several aspects that must be examined. In particular, in the context of short-term serialorder memory, it is an important and debatable matter whether Hebb repetition learning is based on domain-specific or domain-general mechanisms. The vast majority of studies in the field of Hebb repetition learning have been reported for verbal/language domains. The present study examines the Hebb repetition effect in the visuospatial domain with a short-term memory task.

In order to investigate the functioning of the visuospatial short-term memory, the Corsi block task, which requires participants to remember a sequence of spatial locations, is frequently used (e.g., Cornordi \& Vecchi, 2003). It has already been confirmed that the Hebb repetition effects are observed in the visuospatial domain (Couture \& Tremblay, 2006). A remaining question here is whether the Hebb repetition learning in the visuospatial domain is sensitive to variables that are known to affect the verbal Hebb repetition learning.

In the verbal domain, temporal grouping is achieved by inserting temporal pauses between items in a sequence. The temporal grouping effect refers to a better performance of immediate serial recall with grouping than with steady-pace presentation (e.g., Frankish, 1985, 1989; Hitch, Burgess, Towse, \& Culpin, 1996; Ryan, 1969). This phenomenon is not only observed in the verbal domain but also in the visuospatial domain (Hurlstone, 2018; Hurlstone \& Hitch, 2018; Parmentier, Andrés, Elford, \& Jones, 2006). It is of note that when the temporal grouping patterns change in every presentation of the repeated sequences, the Hebb repetition effect is removed (Bower \& Winzenz, 1969) or decreased (Hitch, Flude, \& Burgess, 2009). This negative impact of differential temporal patterns on the Hebb repetition effect was examined and reported only for the verbal domain. Hitch et al. (2009) explained this phenomenon as follows: Short-term retention of serial order is achieved by employment of temporal context that provides timing signals to each item. The Hebb repetition effect reflects the long-term weight changes in the contextitem connection. The long-term connection weights, however, do not change efficiently when the temporal structures vary for each presentation of the repeated sequence. This is because the change in the long-term connection weights can occur only when incoming sequences are matched with representations of sequences in long-term memory through cumulative matching process - similar to the process assumed in the cohort model of auditory word recognition by Marslen-Wilson (1987). If the temporal structures are different, the matching cannot happen, hence the learning does not occur (Burgess \& Hitch, 2006), causing a decline in the Hebb repetition effect in that situation. A chunk-based model of the Hebb repetition effect can also explain this phenomenon (Page \& Norris, 2009). As the chunks implied by the temporal grouping change on each presentation, any given chunk is not well learned due to failure of the cumulative matching between the incoming sequences and the representations of sequence chunks.

In the current study, we examined using a variant of the Corsi block task whether the Hebb repetition learning in the visuospatial domain is sensitive to temporal structures of item sequences. Processing serial-order information might be either a domain-free or a domain-specific ability at the functional level at least to some extent; this study attempts to answer the question of whether the ability to process serial-order information in the visuospatial domain can be explained by the same memory model as in the verbal domain. If the ability to process serial-order information is completely based on domain-general principles, we should expect that the change in temporal structure of the repeated sequence leads to an absence or a decrease of Hebb repetition effects in the visuospatial domain. Before testing this assumption, we investigated in a first experiment how temporal grouping affects Hebb repetition learning. We predicted that the temporal grouping itself facilitates the performance on immediate serial recall of dot sequences (e.g., Hurlstone, 2018; Hurlstone \& Hitch, 2015; Parmentier et al., 2006). A target question is whether temporal grouping increases Hebb repetition learning or whether it has an additive effect on the learning. These results can be informative for the theoretical development of serialorder memory, particularly for theories of the STM-LTM relationship. In addition to this issue, we also examined the participants' awareness of a repeated presentation of the same sequence. As mentioned before, previous studies on the Hebb repetition learning indicate that the effect can occur without such awareness (e.g., Couture \& Tremblay, 2006; Guérard, Saint-Aubin, Boucher, \& Tremblay, 2011). Some recent studies, however, show possible connections between the awareness of repetition and the Hebb repetition effect. The role of awareness in the Hebb repetition learning is an important issue that potentially affects the conceptualization of the domaingeneral or domain-specific nature of long-term sequential learning and that constrains theorization of human memory models. 
We report two experiments in this paper. In Experiment 1, we set two conditions: a grouping condition and a nongrouping condition, and we examined how the temporal grouping effect works under the visuospatial Hebb repetition paradigm. We also examined whether awareness of the repeated sequence affects Hebb repetition learning in this domain. In Experiment 2, there were again two conditions of temporal structures: a random-grouping condition and a fixed-grouping condition. The Hebb list was presented with a different temporal structure in every repetition in the random-grouping condition. We examined whether this manipulation does eliminate or weaken the Hebb effect in the visuospatial domain.

\section{Experiment 1}

In this experiment, we examined how the temporal grouping effect has influences in the visuospatial Hebb repetition paradigm and whether awareness of the repeated sequence affects Hebb repetition learning in this domain. To achieve these aims, we defined two conditions: a grouping condition and a non-grouping condition. They were based on the studies of Couture and Tremblay (2006) and Parmentier et al. (2006). To investigate the effects of awareness on learning, we used the methods of McKelvie (1987) and Couture and Tremblay (2006), in which participants were asked whether they noticed anything particular about the procedure. Responses to this open question informed us whether the participants, who did not have any preconceptions regarding this aspect of the task, had been aware of the repeated sequence. After this question, participants performed a recognition task to test whether they could identify the repeated sequences.

\section{Method}

\section{Participants}

Forty-nine undergraduate and graduate students (27 men and 22 women, Mean $=20.9, S D=1.7$, age range 18-24 years) from Kyoto University participated in this experiment. All of them reported normal or corrected-to-normal vision. They received 500 yen for their participation. One participant was excluded due to an administrative failure, therefore we used data from 48 participants for further analyses.

Half of the participants were randomly assigned to the grouping condition, and the others were assigned to the nongrouping condition.

\section{Stimuli and apparatus}

Stimuli were black dots with a diameter of $1 \mathrm{~cm}$. Nine dots were distributed in a $17 \times 17 \mathrm{~cm}$ square frame, which was drawn with black lines on a white background (see Fig. 1, which shows only the square frame and dots). For each participant, the locations of these nine dots were randomly generated under the condition that the distance between two dots is at least $3 \mathrm{~cm}$. The locations of the nine dots did not change throughout the experiment. In non-repeated sequences, the presentation order of the dots was generated in every trial at random with the additional requirement that not more than two dots were assigned to the same serial position and spatial location they had in the immediately previous trial. In repeated sequences, the presentation order was generated randomly in the fourth trial with the same conditions used in non-repeated sequences, and it was repeatedly presented throughout the experiment in every fourth trial.

For the recognition test, we presented simultaneously four equally sized panels $(8.5 \mathrm{~cm} \times 8.5 \mathrm{~cm}$; see Appendix Fig. 6). Each was a smaller version of the recall test display with white digits displayed on the dots representing their temporal order. One display showed the repeated sequence order (correct display), while others showed random orders (incorrect display). The locations of correct and incorrect displays were assigned randomly across participants.

The task was generated using a Windows 7 computer and MATLAB software with the Psychophysics Toolbox extension (Brainard, 1997; Pelli, 1997; http://psychtoolbox.org/), and all items were presented on a cathode ray tube display (17 in; height, $24 \mathrm{~cm}$; width, $32 \mathrm{~cm}$ ) in a soundproof chamber. The resolution of the display was $1,024 \times 768$ pixels. The distance from the participant's eyes to the display was $50 \mathrm{~cm}$, and the his or her head was fixed using a chin rest. Responses were recorded with an optical mouse and a keyboard.

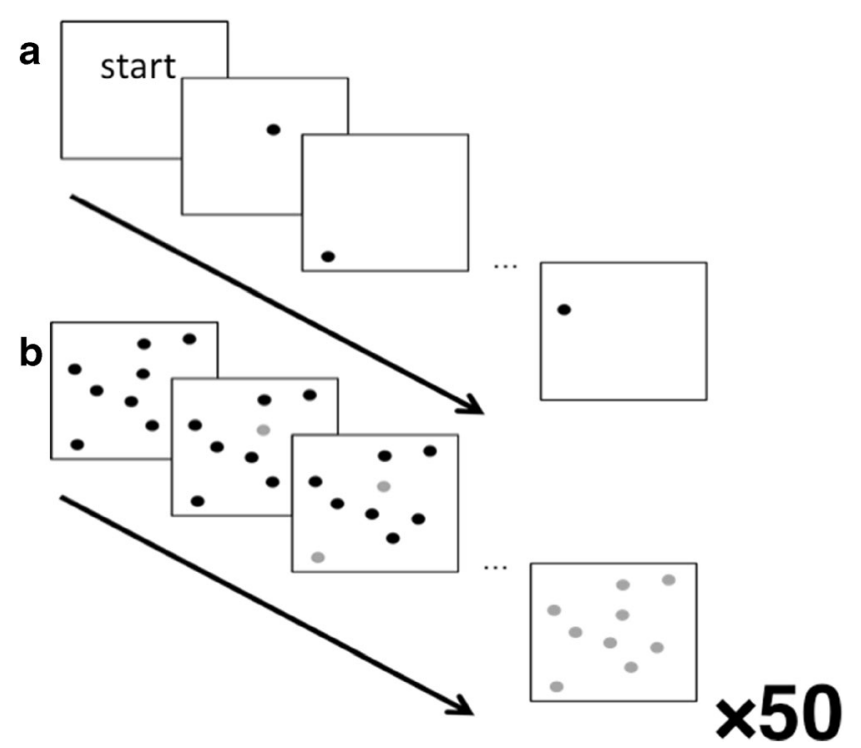

Fig. 1 Schema of the recall task. Each trial consisted of a presentation of nine dots (A) and a subsequent recall test (B). Participants performed 50 trials 


\section{Procedure}

Each participant performed a recall task that was composed of 50 trials and a recognition task.

For the recall task, "start" was presented on the display at the beginning of each trial, and participants were asked to click anywhere to start a trial. After a blank screen for 2,000 ms, nine black dots were presented one by one. In the non-grouping condition, each dot was presented for $500 \mathrm{~ms}$ with a $500-\mathrm{ms}$ inter-stimulus interval, whereas in the grouping condition, the interval after the third and sixth dots was $2,500 \mathrm{~ms}$, indicating that sequences were divided into three groups of three dots each. During this presentation, the black frame remained visible and the mouse pointer invisible (see Fig. 1). After presenting the last dot, only the white background was presented for $500 \mathrm{~ms}$.

Subsequently, the mouse pointer appeared, and the recall test started. In this test, all dots were presented simultaneously (Fig. 1) and participants were asked to click them one by one with a mouse in the same order as they had been presented. When they clicked a dot, the color of it changed from black to gray and could not be chosen again. Participants were also asked to respond as quickly and accurately as possible. Moreover, to avoid encoding the temporal order of only a few dots instead of the whole group, the experimenter encouraged participants to recall all items as accurately as possible. After clicking all dots, the "start" display for the next trial was immediately presented.

In every fourth trial (fourth, eighth, 12th, and so on), the repeated sequences were presented, while in all other trials, the non-repeated sequences were presented. Therefore, the recall task consisted of 12 trials for the repeated sequence and an additional 38 trials for nonrepeated sequences. The last repeated sequence (48th trial) was followed by two non-repeated sequences (49th and 50th trials), which were not used in the analyses. Two practice trials were conducted before starting the experiment. Thus, the number of trials was 52 in total; among those 48 trials were analyzed.

For the recognition task, the experimenter first asked participants "Did you notice anything particular about the procedure?" to evaluate their awareness of repeated sequences after the recall task was finished. If they referred to the repetition, they were classified as "aware of repetition." If they did not, the experimenter then asked them "Did you notice the repetition sequence?", but they were classified as "unaware of repetition" regardless of their answer to the second question. Then, all participants were debriefed that one sequence was repeatedly presented and did the recognition test, in which they had to identify the correct display among the four presented choices (Appendix Fig. 6).

\section{Results}

We excluded the data of two participants - one in the grouping and one in the non-grouping condition - from further analyses because their mean numbers of correct recalls (maximum $=9$ ) were very low (1.75 and 2.06, respectively, which were lower than the Mean $-2 S D$ of other participants in their respective group). Therefore, analyses used 23 participants in the grouping condition and 23 participants in the non-grouping condition.

We summarized the recall task into three epochs, following Couture and Tremblay (2006): from the first to 16th trials as the first epoch, from the 17th to 32nd trials as the second epoch, and from the 33rd to 48th trials as the third epoch. Sequences presented three, two, and one trial before the repeated sequence were called nonrepeated1, nonrepeated2, and nonrepeated3, respectively. Each sequence was presented 12 times, thus four trials from each repetition type were in each epoch.

\section{Proportion of correct serial recall}

Figure 2 shows the proportion of correct serial recall in Experiment 1. A 2 (grouping: grouping and non-grouping; between-participants) $\times 3$ (epoch: within-participant) $\times 4$ (repetition: nonrepeated1, nonrepeated2, nonrepeated3, and repeated; within-participant $) \times 9$ (serial position; within-participant) ANOVA was conducted on the data of proportion of correct serial recall. First, the main effect of grouping was significant $\left[F(1,44)=7.10, p=.011, \eta_{\mathrm{p}}^{2}=.139\right]$, indicating that the temporal grouping effect occurred: Mean proportion of correct recall was higher in the grouping condition (.65) than in the non-grouping condition (.55). Second, the interaction between grouping and serial position was significant $[F(8,352)=5.47, p$ $\left.<.001, \eta_{\mathrm{p}}{ }^{2}=.111\right]$. Subsequent analyses revealed that the recall performance improved by temporal grouping at the second, third, fourth, fifth, sixth, and seventh serial positions in the temporal grouping condition. However, the serial position analysis for the grouping condition revealed that there were no significant differences between the first and second, fourth and fifth, and seventh and eighth serial positions. This indicates that the temporal grouping improved the recall performances from the second to the seventh serial positions, but the serial position curves did not show the shapes that were observed in the temporal grouping condition with verbal materials - three mini serial position curves within a nine-item list (e.g., Frankish, 1985). Third, the three-way interaction among epoch, repetition, and serial position was significant $[F(48,2112)=$ $\left.1.49, p=.018, \eta_{\mathrm{p}}^{2}=.033\right]$. Subsequent analyses showed that the effect of repetition was not observed in the first epoch, whereas it was observed from the fourth to the ninth serial positions in the second and third epochs. Fourth, the interaction between grouping, epoch and repetition was not significant $\left[F(3,132)=0.47, p=.705, \eta_{\mathrm{p}}^{2}=.027\right]$. This suggests that the temporal grouping did not affect the Hebb repetition effect. 

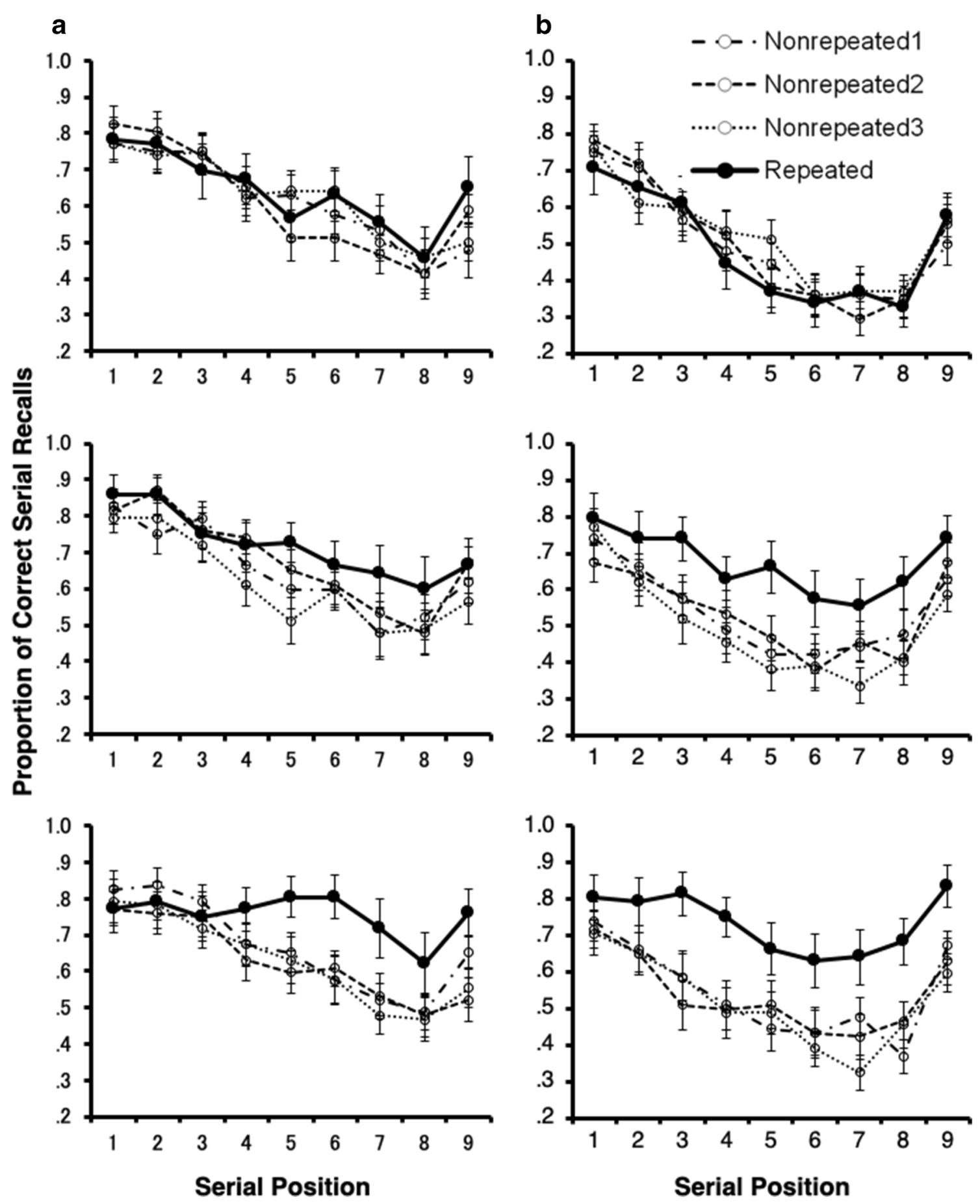

Fig. 2 Proportion of correct serial recall in the grouping condition (A) and the non-grouping condition (B) of Experiment 1. The top, middle,

\section{Awareness}

Twenty-three participants $(50.0 \%)$ were aware of the repetition (12 of them [52.2\%] were in the grouping condition), and 21 of them $(91.3 \%)$ could correctly recognize the repeated sequence. On the other hand, 23 participants were not aware of the repetition (11 of them [47.8\%] were in the grouping condition), and 16 of them $(69.6 \%)$ could answer correctly in the recognition test.

and bottom row show the first, second, and third epochs, respectively. Error bars indicate standard errors

\section{Relation between the proportion of correct serial recall} and awareness

As a post hoc analysis, we evaluated the relation between the proportion of correct serial recall and awareness. Figure 3 shows the proportion of correct recall as a function of grouping, awareness, repetition, and epoch. A 2 (grouping: grouping and non-grouping; between-participants) $\times 2$ (awareness; 
between-participants) $\times 4$ (repetition: nonrepeated 1 , nonrepeated 2 , nonrepeated 3 , and repeated; within-participant) $\times 3$ (epoch; within-participant) ANOVA was conducted on the data of the proportion of correct serial recall. First, the main effect of grouping was significant $[F(1,42)=7.56, p=.009$, $\left.\eta_{\mathrm{p}}{ }^{2}=.152\right]$, but all of the interactions related to grouping were not significant $\left[F s<1.35, p s>.237, \eta_{\mathrm{p}}{ }^{2}<.031\right]$. This suggests that the grouping affected just the whole performance regardless of awareness. Second, the interaction among awareness, repetition, and epoch was significant $[F(6,252)=2.36, p=$ $\left..031, \eta_{\mathrm{p}}{ }^{2}=.053\right]$. Subsequent analyses indicate that the Hebb repetition effect was observed in the second and third epochs only in participants who were aware of repetition.

\section{Relation between repetition-induced improvement and awareness}

To recast the correct recall into the gradient of improvement, the correct recall was linearly regressed as a function of epochs for each participant. A 2 (grouping: grouping and non-grouping; between-participants) $\times 2$ (awareness; between-participants) $\times$
4 (repetition: nonrepeated1, nonrepeated2, nonrepeated3, and repeated; within-participant) ANOVA was conducted on the gradients of improvement. First, the main effect of repetition was significant $\left[F(3,126)=9.21, p<.001, \eta_{\mathrm{p}}{ }^{2}=.180\right]$. The gradient (i.e., learning slope) is larger for the repeated sequences (.811) than non-repeated sequences $(.223, .117$, and .014 for nonrepeated1, nonrepeated2, and nonrepeated3, respectively). Second, the main effect of grouping was not significant $[F(1$, $\left.42)=0.943, p=.337, \eta_{\mathrm{p}}{ }^{2}=.022\right]$. This indicates that grouping did not affect the gradients of improvement (i.e., the size of the learning). Third, the interaction between awareness and repetition was significant $\left[F(3,126)=3.90, p=.011, \eta_{\mathrm{p}}{ }^{2}=.085\right]$. Subsequent analyses indicate that repeated sequences (gradient $=.976)$ showed larger improvements than any non-repeated sequence $(-.118, .247$, and .041 for nonrepeated1, nonrepeated2, and nonrepeated 3 , respectively) in participants who were aware of repetition, whereas in participants who were unaware of repetition, repeated sequences (.645) showed larger improvements than nonrepeated 2 (-.013) and nonrepeated3 sequences (-.013), but did not differ substantially from nonrepeated 1 sequences (.565). These results suggest that the performance
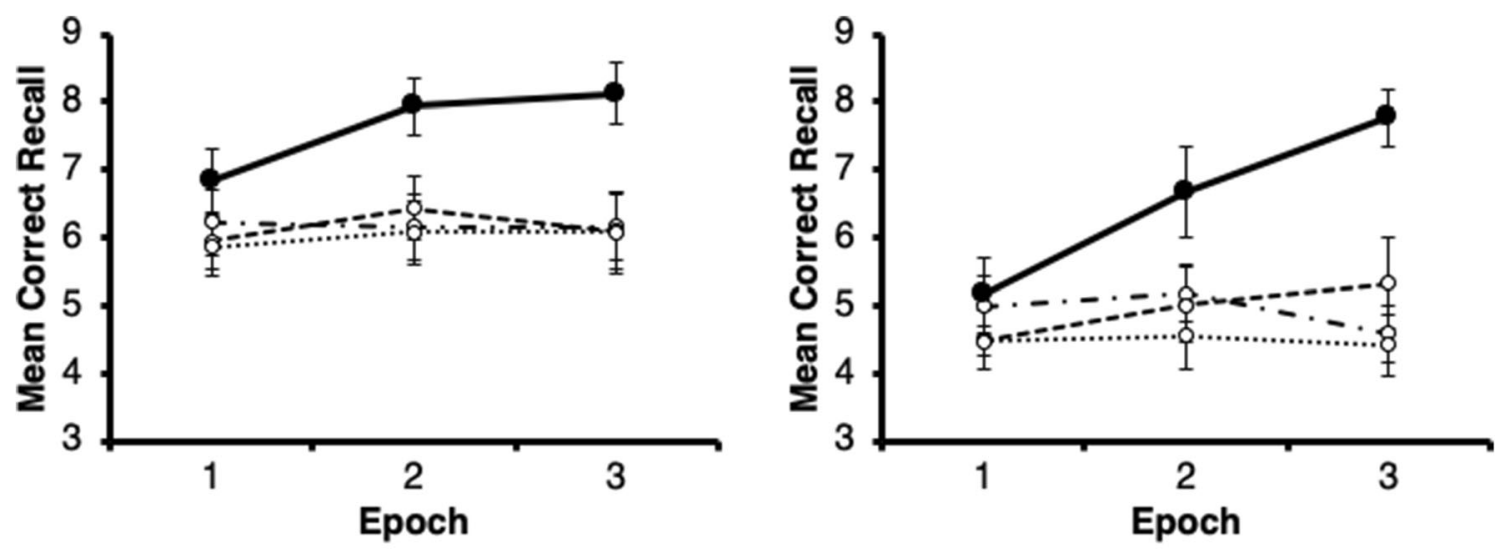

Aware, Grouping

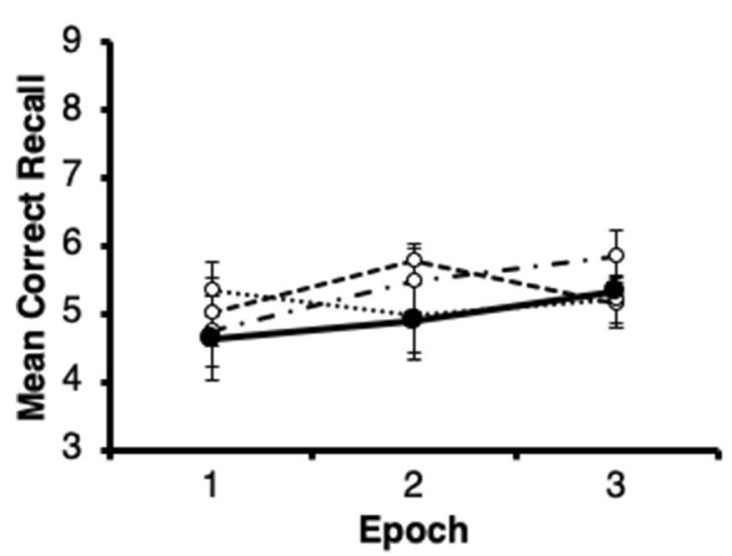

Unaware, Grouping

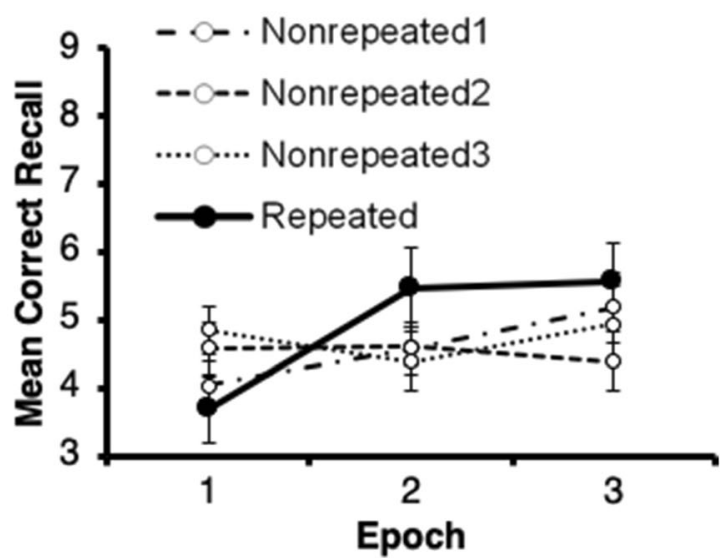

Unaware, Non-grouping

Fig. 3 Relation between the proportion of correct serial recall and awareness in Experiment 1 
improvements in the repeated sequences were larger than those in some of the filler sequences even in participants who were not aware of the repetition, that is, the Hebb repetition effect was observed not only in participants who were aware of repetition but also in those unaware of it.

\section{Reaction times}

Reaction time data from Experiment 1 are shown in Appendix Fig. 7. A 2 (grouping: grouping and non-grouping; betweenparticipants $) \times 3$ (epoch; within-participant) $\times 4$ (repetition: nonrepeated1, nonrepeated 2 , nonrepeated3, and repeated; within-participant) $\times 9$ (serial position; within-participant) ANOVA was conducted on the data of the reaction time of the recall test. First, the interaction between grouping and serial position was significant $\left[F(8,352)=3.05, p=.003, \eta_{\mathrm{p}}{ }^{2}=.065\right]$. In the subsequent analyses, we found significant differences between the third and fourth positions and the fourth and fifth positions in the grouping condition. These suggest that participants divided sequences in accordance with the difference of the interstimulus intervals. However, we did not find significant differences between the sixth and seventh positions and the seventh and eighth positions in the grouping condition. Second, the interaction between epoch and serial position was significant $\left[F(16,704)=2.02, p=.010, \eta_{\mathrm{p}}{ }^{2}=.044\right]$. Subsequent analyses indicate that reaction times from the first to the sixth serial positions were shorter in the second and third epochs than in the first epoch, except the comparison at the third serial position between the first epoch and the third epoch.

\section{Discussion}

The results of this experiment can be summarized in the following three categories. First, the interaction between temporal grouping and repetition was not found although both effects were present. This indicates that the temporal grouping effect did not affect the Hebb repetition effect. Second, the proportion of correct recall looked like a typical serial position curve (Fig. 2) even in the grouping condition, notably, those in the fourth and seventh serial positions - which were groupinitial items - were not significantly higher than those in the fifth and eighth serial positions, respectively, in the grouping condition. Thus, mini serial position curves, which are typically observed in the verbal temporal grouping experiments, were not observed here. This tendency is similar to the results observed in Parmentier et al. (2006), but different to those in Hurlstone (2018) and Hurlstone and Hitch (2015), which showed mini within-group primacy and recency effects. We return to this issue in the General discussion. Third, the Hebb repetition effect was only observed in participants who were aware of repetition when analyzing the proportion of correct serial recall. However, the results of this experiment also showed that the gradient of performance improvement in the
Hebb lists was larger than in the non-repeated lists even in participants who were unaware of repetition, suggesting that not only explicit learning occurred. This finding is in agreement with that of Couture and Tremblay (2006) on Hebb repetition learning in the visuospatial domain. The possible interpretation of this finding is discussed in the General discussion section.

\section{Experiment 2}

In Experiment 2, there were two conditions of temporal structures: a random-grouping condition and a fixed-grouping condition. In the random-grouping condition, we varied the temporal grouping in every trial and investigated whether this manipulation influences Hebb repetition learning and awareness of the repetition. In the fixed-grouping condition, temporal grouping structures varied across participants, but each participant received a respective fixed temporal grouping pattern.

\section{Method}

\section{Participants}

Forty-nine undergraduate and graduate students (25 men and 24 women, Mean $=21.1, S D=2.6$, range 18-34 years) from Kyoto University participated in this experiment. All of them reported normal or corrected-to-normal vision. They received 500 yen for their participation. One participant was excluded due to an administrative mistake, thus we used the data of 48 participants for further analyses.

Half of the participants were randomly assigned to the random-grouping condition, and all other participants were assigned to the fixed-grouping condition.

\section{Stimuli and apparatus}

The stimuli and apparatus were identical to those used in Experiment 1.

\section{Procedure}

For each trial in the random-grouping condition, two interstimulus intervals were randomly selected among the eight intervals, and an interval duration of 2,500 $\mathrm{ms}$ instead of $500 \mathrm{~ms}$ was inserted there. In the first trial of the fixedgrouping condition, two inter-stimulus intervals were randomly selected among the eight intervals, and an interval duration of 2,500 ms was inserted there. This temporal grouping structure was assigned to each participant and fixed throughout the experiment in the fixed-grouping condition. All other procedural details were the same as in Experiment 1. 


\section{Results}

We removed data from one participant in the fixed-grouping condition because his/her mean correct recall was very low (2.69, which was lower than the Mean - 2SD of the other participants in that condition). Therefore, analyses used data from 24 participants in the random-grouping condition and those from 23 participants in the fixed-grouping condition. We divided the recall task into three epochs as in Experiment 1 .

\section{Proportion of correct serial recall}

Figure 4 shows the proportion of correct serial recall in Experiment 2. A 2 (grouping: random-grouping and fixedgrouping; between-participants) $\times 3$ (epoch; withinparticipant $) \times 4$ (repetition: nonrepeated 1 , nonrepeated 2 , nonrepeated3, and repeated; within-participant) $\times 9$ (serial position; within-participant) ANOVA was conducted on the data of the proportion of correct serial recall. First, the main effect of grouping was significant, indicating the higher performance in the fixed-grouping (.62) than in the randomgrouping condition $(.53)\left[F(1,45)=8.27, p=.006, \eta_{\mathrm{p}}{ }^{2}=\right.$ $.155]$, whereas all of the interactions including the grouping factor were not significant $\left[F s<1.28, p s>.164, \eta_{\mathrm{p}}{ }^{2}<.028\right]$. This suggests that grouping affected the whole performance but did not have an influence on the Hebb repetition effect. Second, the interaction between epoch and repetition was significant $\left[F(6,270)=7.64, p<.001, \eta_{\mathrm{p}}^{2}=.145\right]$. Subsequent analyses indicate that the Hebb repetition effect was not observed in the first epoch, whereas it was observed in the second and third epochs. Third, the main effect of serial position was significant $\left[F(8,360)=67.39, p<.001, \eta_{\mathrm{p}}{ }^{2}=.600\right]$. However, all of the interactions related to serial position were not significant $\left[F_{S}<1.64, p s>.053, \eta_{\mathrm{p}}{ }^{2}<.035\right]$.

\section{Awareness}

Twenty-five participants (53.2\%) were aware of the repetition (nine of them [36.0\%] were in the random-grouping condition), and 24 of them (96.0\%) could correctly recognize the repeated sequences. On the other hand, 22 participants were not aware of the repetition (15 of them [68.2\%] were in the random-grouping condition) with 15 of them $(68.2 \%)$ correctly recognizing the repeated sequences.

\section{Relation between the proportion of correct serial recall and awareness}

As a post hoc analysis, we examined the relation between the proportion of correct serial recall and awareness. Figure 5 shows the proportion of correct recall as a function of grouping, awareness, repetition, and epoch. A 2 (grouping: random- grouping and fixed-grouping; between-participants $) \times 2$ (awareness; between-participants) $\times 4$ (repetition: nonrepeated 1 , nonrepeated 2 , nonrepeated 3 , and repeated; within-participant) $\times 3$ (epoch; within-participant) ANOVA was conducted on the data of the proportion of correct serial recall. First, the main effect of grouping was not significant $[F(1,43)=$ $\left.3.33, p=.075, \eta_{\mathrm{p}}{ }^{2}=.072\right]$, and all of the interactions including grouping were not significant $\left[F_{s}<2.93, p s>.059, \eta_{\mathrm{p}}{ }^{2}<.064\right]$. Second, the interaction between awareness, repetition, and epoch was not significant $\left[F(6,258)=0.83, p=.548, \eta_{\mathrm{p}}{ }^{2}=.019\right]$. Third, the interaction between repetition and epoch was significant $\left[F(6,258)=5.49, p<.001, \eta_{\mathrm{p}}^{2}=.113\right]$. The Hebb repetition effect was observed in the second and third epochs but not in the first epoch. Fourth, the main effect of awareness was significant $\left[F(1,43=14.88, p<.001), \eta_{\mathrm{p}}^{2}=.257\right]$.

\section{Relation between repetition-induced improvement and awareness}

A 2 (grouping: random-grouping and fixed-grouping; between-participants) $\times 2$ (awareness; between-participants) $\times$ 4 (repetition: nonrepeated1, nonrepeated2, nonrepeated3, and repeated; within-participant) ANOVA was conducted on the data of the gradients of improvement. The main effect of grouping was not significant $\left[F(1,43)=0.06, p=.814, \eta_{\mathrm{p}}{ }^{2}=\right.$ $.001]$, but the main effect of repetition was significant $[F(3$, 129) $\left.=11.76, p<.001, \eta_{\mathrm{p}}{ }^{2}=.215\right]$. The gradient is larger for the repeated sequences $(.727)$ than non-repeated sequences (-.092, .031, and .055 for nonrepeated1, nonrepeated2, and nonrepeated 3 , respectively). The interaction among grouping, awareness, and repetition was significant $[F(3,129)=3.52, p$ $\left.=.017, \eta_{\mathrm{p}}^{2}=.075\right]$. Subsequent analyses indicate that although the interaction between awareness and repetition was significant in the fixed-grouping condition $[F(3,129)=4.11$, $\left.p=.008, \eta_{\mathrm{p}}{ }^{2}=.136\right]$, the interaction between awareness and repetition was not significant in the random-grouping condition $\left[F(3,129)=0.96, p=.412, \eta_{\mathrm{p}}^{2}=.053\right]$. The other subsidiary analyses related to the above two subsequent analyses were non-significant.

\section{Reaction time}

Reaction time data from Experiment 2 are shown in Appendix Fig. 8. A 2 (grouping: random-grouping and fixed-grouping; between-participants) $\times 3$ (epoch; withinparticipant $) \times 4$ (repetition: nonrepeated1, nonrepeated 2 , nonrepeated3, and repeated; within-participant) $\times 9$ (serial position; within-participant) ANOVA was conducted on the data of the reaction time in the recall test. First, the interaction between repetition and serial position was significant $\left[F(24,1080)=2.42, p<.001, \eta_{\mathrm{p}}{ }^{2}=.051\right]$. Subsequent analyses confirmed that the reaction time in repeated sequences was faster than all of non-repeated sequences only at the first 

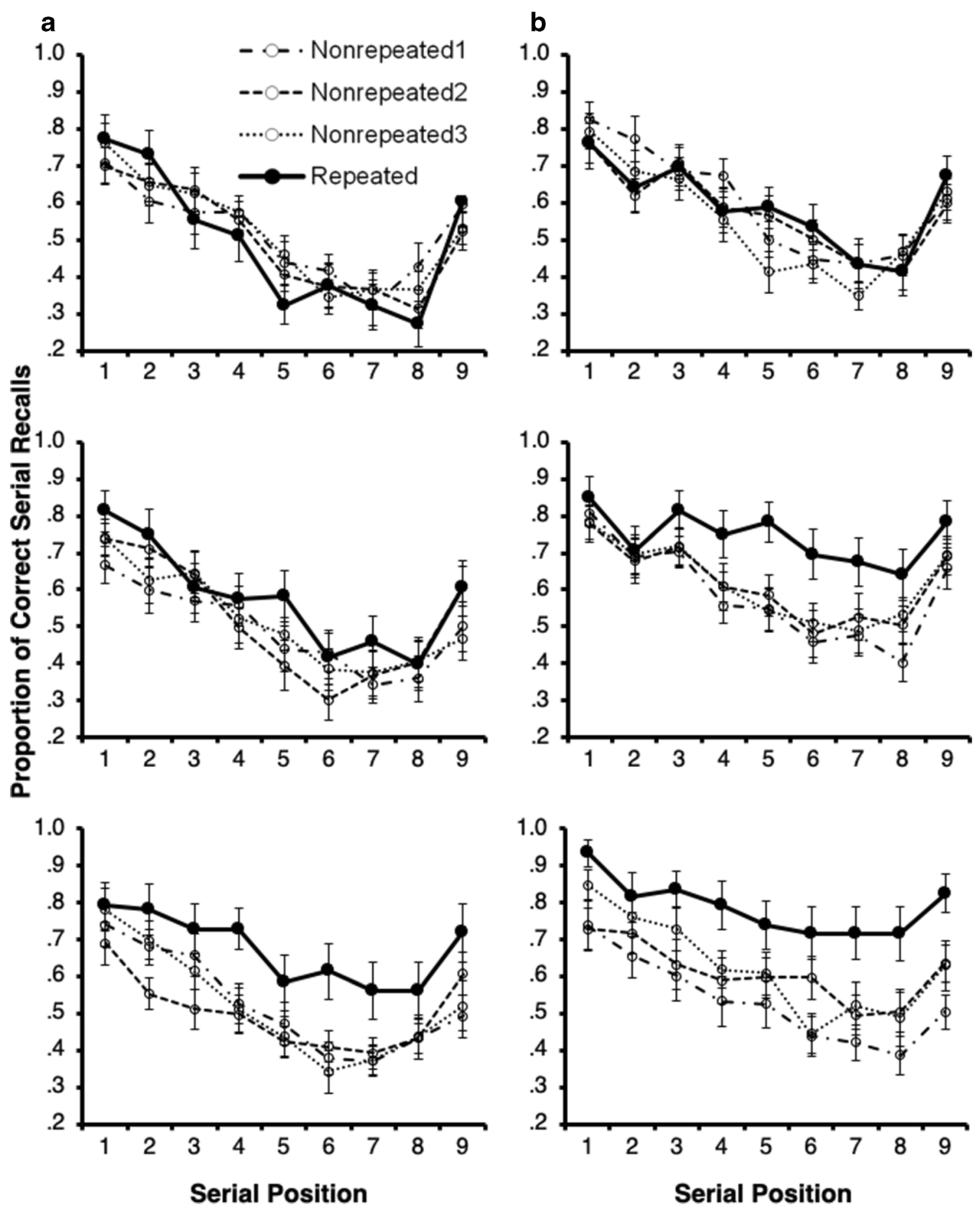

Fig. 4 Proportion of correct serial recall in the random-grouping condition (A) and fixed-grouping condition (B) of Experiment 2. The top,

serial position. Second, the interaction among grouping, epoch, and serial position was significant $[F(16,720)=1.87, p$ $\left.=.021, \eta_{\mathrm{p}}{ }^{2}=.040\right]$. Subsequent analyses indicate that the reaction time was significantly longer in the fixed-grouping condition than in the random-grouping condition only at the first serial position of the first epoch, but this difference immediately disappeared. middle, and bottom rows show the first, second, and third epochs, respectively. Error bars indicate standard errors

\section{Discussion}

We observed both the effect of timing manipulation and the Hebb repetition effect. However, the interaction between them was not significant, suggesting that changes in temporal structures of the to-be-remembered lists did not affect the Hebb repetition effect. This is consistent with the results of 


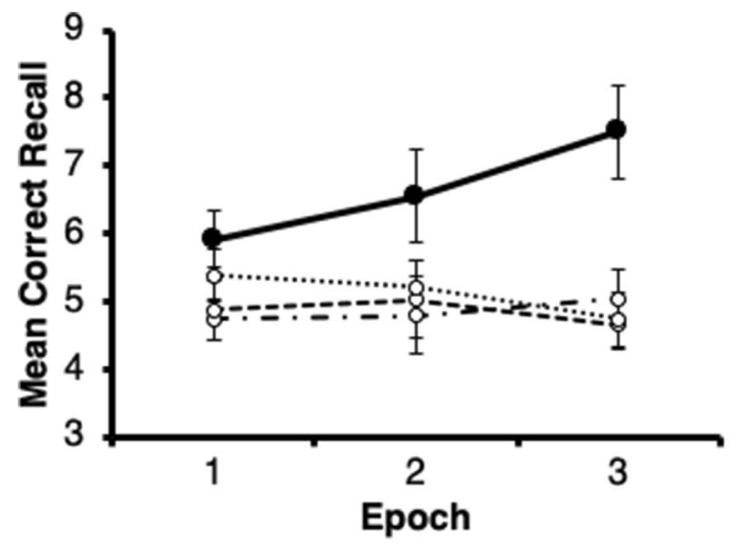

Aware, Random-grouping

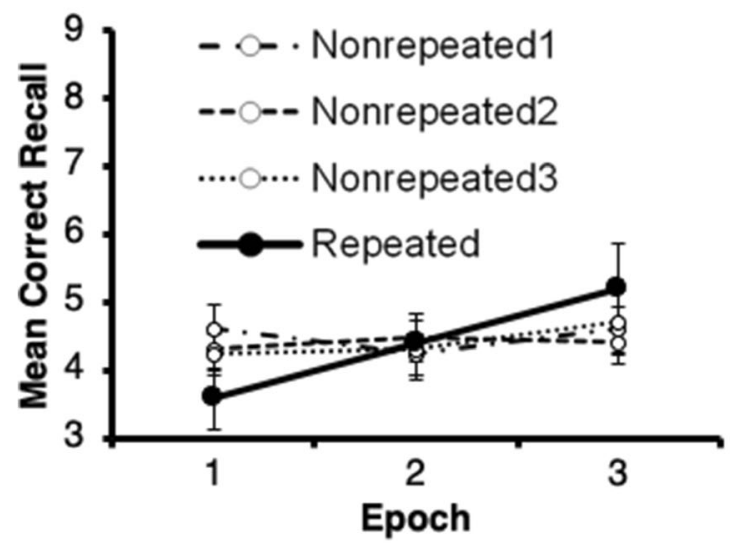

Unaware, Random-grouping

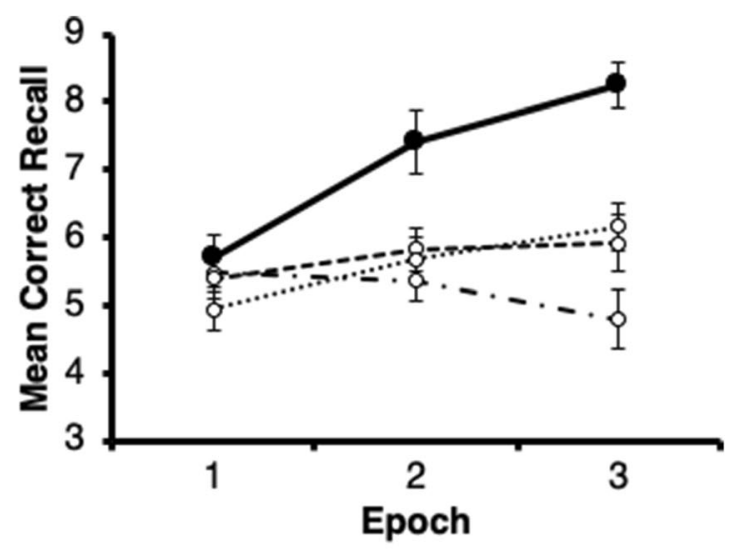

Aware, Fixed-grouping

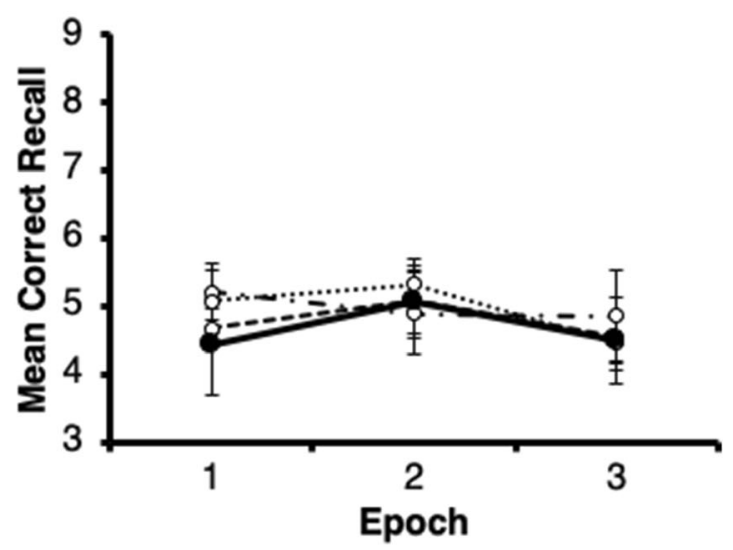

Unaware, Fixed-grouping

Fig. 5 Relation between the proportion of correct serial recall and awareness in Experiment 2

Experiment 1. In the verbal domain, the Hebb repetition effect became very weak when the temporal grouping was changed in each trial, indicating that Hebb repetition learning is strongly linked to the temporal context (Hitch et al., 2009). The results of this experiment were different from those of the previous studies in the verbal domain.

The statistical results of the analyses with awareness of repetition were equivocal. However, the results of this experiment at least preserve the possibility that implicit learning occurred in the random-grouping condition.

\section{General discussion}

Hebb repetition effects in the visuospatial domain were examined in two short-term memory experiments. The results are summarized as follows: First, the presentation timing manipulations did not interact with the Hebb repetition effect. Although both the temporal grouping effect and the Hebb repetition effect were clearly replicated, the interaction between them was not found in Experiment 1. Similarly, although both the disruptive effect by presentation timing changes and the facilitative effect by Hebb repetition learning were observed, the interaction between them was not detected in Experiment 2. Second, the proportion of correct recall at the fourth and seventh serial positions, which were the group-initial items of the second and third groups within a list respectively, were not significantly higher than those at the fifth and eighth serial position, resulting in serial position curves that were more characteristic of ungrouped lists. Third, the Hebb repetition effect was clearly observed in participants who were aware of repetition while the weak effect was confirmed in those who were not aware of repetition in the analyses of learning gradients. These three issues are discussed in the order listed.

In Experiment 1, the temporal grouping did not affect the Hebb repetition effect. In the verbal domain, the temporal grouping effect on Hebb repetition learning has not been properly tested thus far. In one study investigating this topic, Smalle et al. (2016) tested the Hebb repetition effect under a temporal grouping of small chunks (grouping by two consonant/vowel syllables; Smalle et al., 2016; Experiment 3), and this grouping paradigm made adult participants behave like children. In that 
condition, a larger Hebb repetition effect is observed when the items used in repeated sequences are different from the ones used in non-repeated sequences (the non-overlap condition) in comparison to experiments in which repeated and non-repeated sequences share the same items (the overlap condition). More importantly, the grouping manipulation leads to a larger Hebb repetition effect in the non-overlap than in the overlap condition. This suggests that chunking by temporal pauses of sequences is beneficial for Hebb repetition learning in the verbal domain only when items do not overlap between repeated sequences and non-repeated sequences. In the current study, we presented the dots at the same locations throughout the experiment for each participant, conceptually similar to the overlap condition in Smalle et al. (2016). Therefore, it is not surprising that chunking by temporal pauses did not have a positive effect on Hebb repetition learning in our experiment. We must, however, draw attention to the fact that in Smalle et al. (2016) temporal grouping did not improve the entire memory performance in the filler lists. This means that the standard temporal grouping effect did not appear in Experiment 3 of Smalle et al., suggesting the necessity for a careful interpretation of their data.

The disruptive effect of differential temporal structures on Hebb repetition learning was observed in an experiment with digit lists where both repeated and non-repeated sequences consisted of digits, that is, in the overlap condition (Hitch et al., 2009). In Experiment 2 of the current study, we did not find this disruptive effect. It is possible that visuospatial sequential learning might be less sensitive to temporal structures than verbal sequential learning, although temporal grouping and presentation timing changes had a beneficial effect and a disruptive effect, respectively, on the immediate serial recall of dot sequences in the present study.

A recent short-term memory study on temporal grouping effects provides a clue to understanding the differential nature of verbal and spatial sequential learning. Hurlstone (2018) indicated that short-term verbal serial order memory uses representations of group positions in a sequence and item positions within a group whereas short-term spatial serial order memory holds representations of group positions in a sequence and item positions in a WHOLE sequence. It is assumed that representations of item positions in a sequence is less sensitive to the disruption of temporal grouping than representations of item positions within a group because the former relies less on grouping structures than the latter. This assumption predicts the pattern of the results observed in the current study and support the idea that positional representations in verbal and visuospatial short-term memory are underpinned by domainspecific mechanisms (see, Hurlstone, 2018).

The proportion of correct recall in the initial position of each grouping was not significantly higher than the next two positions although it has been reported for the verbal domain that mini serial position curves (with higher performance at the fourth and seventh serial positions) emerge for temporal grouping lists particularly with auditory item presentation (e.g., Frankish, 1989). A similar pattern of data was reported in a previous study (Parmentier et al., 2006), that is, shapes of mini serial position curves for visuospatial materials are not remarkable in the temporal grouping condition. However, Hurlstone (2018) and Hurlstone and Hitch (2015) reported within-group primacy and recency effects in the temporal grouping condition of spatial short-term memory experiments. A clear methodological difference between the former (the current study; Parmentier et al., 2006) and the latter (Hurlstone, 2018; Hurlstone \& Hitch, 2015) is in the recall procedures. In the former studies, when participants clicked a dot during a recall phase, the color of the selected dot changed from black to gray. This means that a dot selected once could not be chosen again. In contrast, the color of the selected location changed temporarily and returned to the original color again in the latter studies, meaning that each location could be selected more than once. Note that most experiments in verbal short-term memory employ oral or written serial recall tasks in which the same items can potentially be selected in one trial. Although it is not clear whether such a methodological difference can generate the differential patterns in serial position curves, the opportunities to select the same item again allow participants to choose an item independently from previous choices, raising potential sensitivities to position-item associations.

Regarding the third point, awareness of repetition, previous studies (e.g., McKelvie, 1987) indicate that the Hebb repetition learning is implicit learning. Nevertheless, in this study, awareness seems to be associated with a performance increase through the repetition of sequences. The recall performance of participants who were aware of repetition was much higher than that of participants who were unaware of repetition. We should, however, note that the analyses of the repetitioninduced improvements (the learning gradient) indicate that learning improvement for the Hebb repetition sequence was higher than that of some filler sequences even if participants were not aware of repetition, suggesting that some sort of implicit learning might have operated in the visuospatial Hebb repetition learning. This conclusion is consistent with the results reported in Couture and Tremblay (2006), which demonstrated that participants who are aware of repetition show a higher overall memory performance in a visuospatial task than those who are not, but learning repeated sequences is more improved than filler sequences regardless of awareness. The fact that the aware participants show a higher memory performance overall indicates that they were aware of the repetition because they remember the sequences well (specifically, this tendency was strongly observed in the fixed temporal grouping of our Experiment 2). This is a plausible explanation and the awareness itself might not have promoted Hebb repetition learning. This is another possibility in the relationship between memory performance and awareness 
of the repetition and provides us with an opportunity to rethink the relationship between STM and LTM. The causal relationship between awareness of repetition and the Hebb repetition learning should be systematically examined for further understanding of the STM-LTM relationship.

\section{Author Note}

Momoe SUKEGAWA is now at the Graduate School of Biostudies, Kyoto University.

This research was financially supported in part by JSPS KAKENHI Grant Number 16K04424 to SS. We would like to thank Kaichi Yanaoka, Kyoto University, for numerous useful discussions and Tomohiro Nobeyama, Toyama Prefectural University, for helpful advice on the manuscript. We are also grateful to Mike Page and Mark Hurlstone for thier constructive comments on an earlier version of the manuscript of this paper.

\section{Appendix 1}

Schema of recognition test
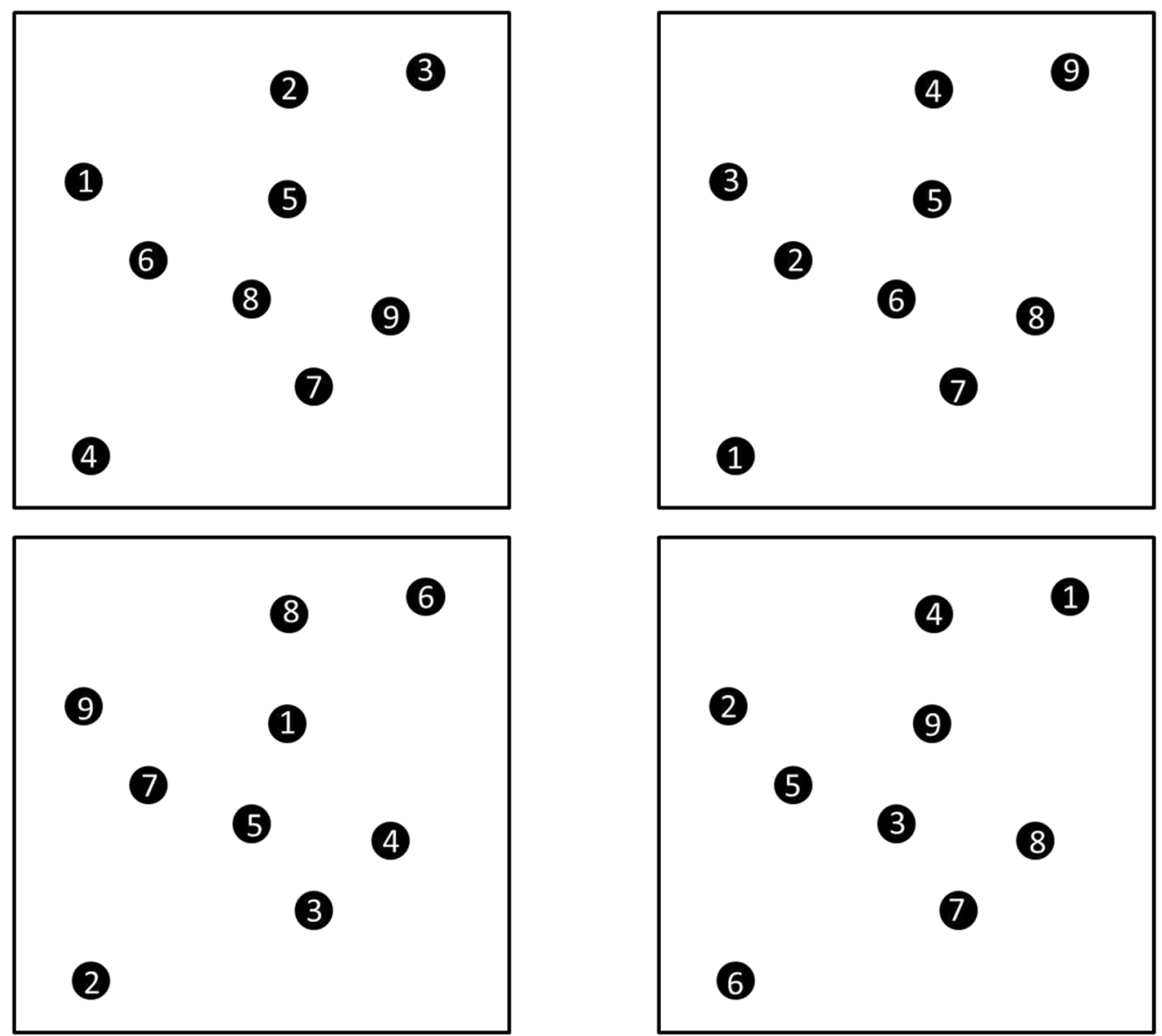

Fig. 6 Schema of the recognition test. Participants were asked to select the panel displaying the correct repeated sequence order 


\section{Appendix 2}

Reaction time of Experiment 1

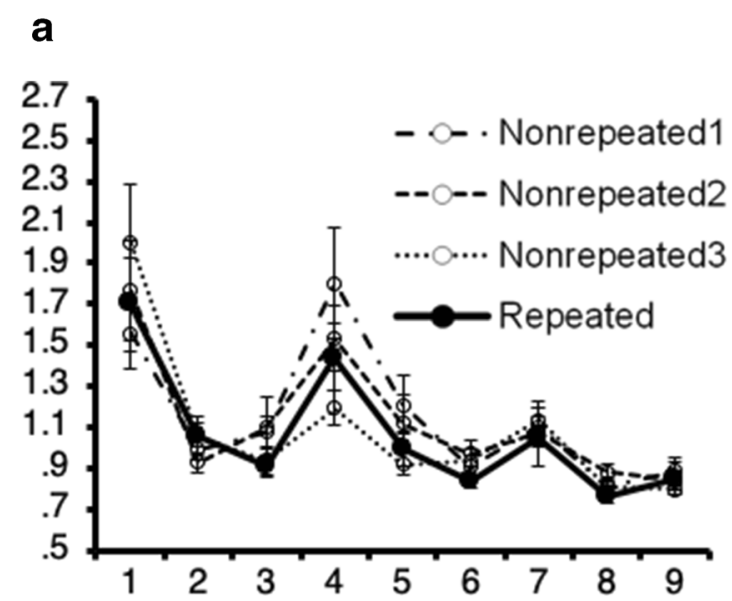

b
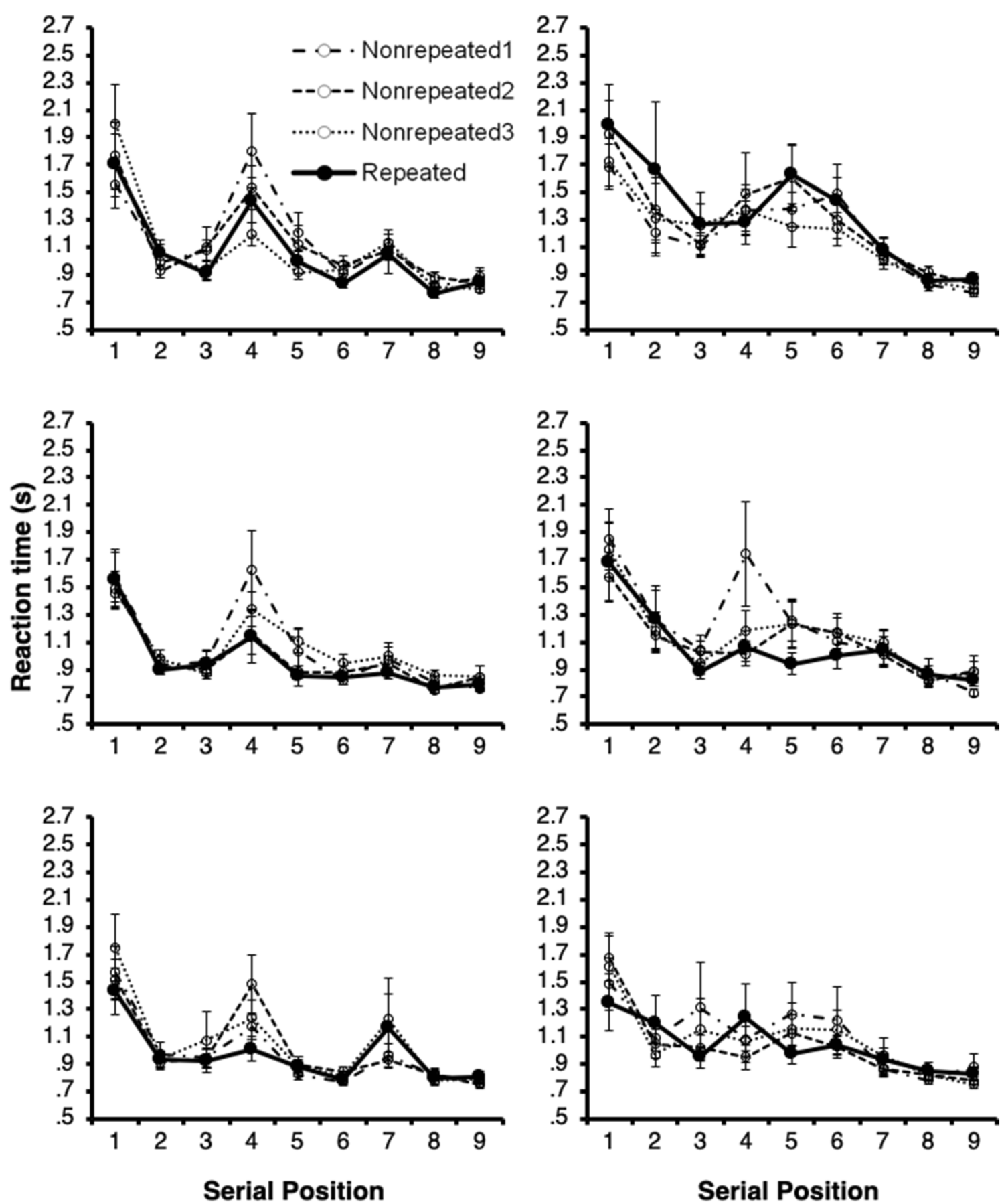

Fig. 7 Reaction time in the grouping condition (A) and the non-grouping condition (B) of Experiment 1. The top, middle, and bottom rows show the first, second, and third epochs, respectively. Error bars indicate standard errors 


\section{Appendix 3}

Reaction time of Experiment 2
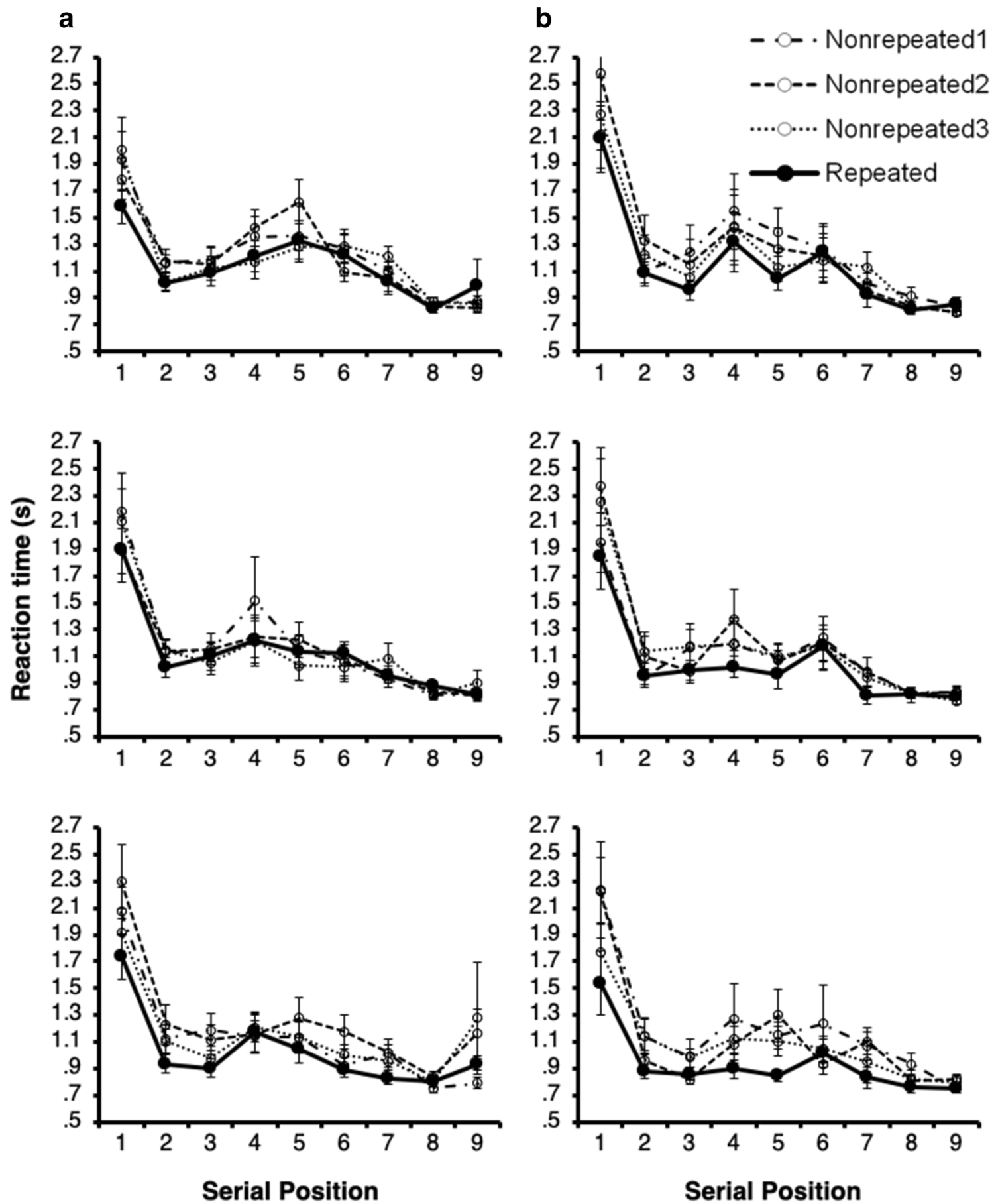

Fig. 8 Reaction time in the random-grouping condition (A) and fixed-grouping condition (B) of Experiment 2. The top, middle, and bottom rows show the first, second, and third epochs, respectively. Error bars indicate standard errors 


\section{References}

Atkinson, R. C., \& Shiffrin, R. M. (1968). Human memory: A proposed system and its control processes. In K. W. Spence \& J. T. Spence (Eds.), The psychology of learning \& motivation: Advances in research \& theory (Vol. 2, pp. 89-195). New York: Academic Press.

Baddeley, A.D., Gathercole, S.E., Papagno, C. (1998). The phonological loop as a language learning device. Psychological Review, 105, 158-173.

Bower, G. H., \& Winzenz, D. (1969). Group structure, coding, and memory for digit series. Journal of Experimental Psychology Monograph, 80, 1-17.

Brainard, D. H. (1997). The Psychophysics Toolbox. Spatial Vision, 10, 433-436.

Burgess, N., \& Hitch, G. J. (2006). A revised model of short-term memory and long-term learning of verbal sequences. Journal of Memory and Language, 55, 627-652.

Cornordi, C., \& Vecchi, T. (2003). Visuo-spatial working memory and individual differences. Hove: Psychology Press.

Couture, M., \& Tremblay, S. (2006). Exploring the characteristics of the visuospatial hebb repetition effect. Memory \& Cognition, 34, 17201729.

Frankish, C. R. (1985). Modality-specific grouping effects in short-term memory. Journal of Memory and Language, 24, 200-209.

Frankish, C. R. (1989). Perceptual organization and PAS. Journal of Experimental Psychology: Human Learning and Memory, 15, 469-479.

Guérard, K., Saint-Aubin, J., Boucher, P., and Tremblay, S. (2011). The role of awareness in anticipation and recall performance in the Hebb repetition paradigm: implications for sequence learning. Memory \& Cognition, 39, 1012-1022.

Hebb, D. O. (1961). Distinctive features of learning in the higher animal. In J. F. Delafresnaye (Ed.), Brain mechanisms and learning (pp. 3746). New York: Oxford University Press.

Hitch, G. J., Burgess, N., Towse, J. N., \& Culpin, V. (1996). Temporal grouping effects in immediate recall: A working memory analysis. Quarterly Journal of Experimental Psychology, 49A, 140-158.

Hitch, G. J., Flude, B., \& Burgess, N. (2009). Slave to the rhythm: Experimental tests of a model for verbal short-term memory and long-term sequence learning. Journal of Memory and Language, 61, 97-111.

Hurlstone, M. J. (2018). Functional similarities and differences between the coding of positional information in verbal and spatial short-term order memory. Memory https://doi.org/10.1080/09658211.2018. 1495235
Hurlstone, M. J., \& Hitch, G. J. (2015). How is the serial order of a spatial sequence represented? Insights from transposition latencies. Journal of Experimental Psychology: Learning, Memory, and Cognition, 41(2), 295-324.

Hurlstone, M. J., \& Hitch, G. J. (2018). How is the serial order of a visual sequence represented? Insights from transposition latencies. Journal of Experimental Psychology: Learning, Memory, and Cognition, 44(2), 167-192.

Hurlstone, M. J., Hitch, G. J., \& Baddeley, A. D. (2014). Memory for serial order across domains: An overview of the literature and directions for future research. Psychological Bulletin, 140, 339-373.

Lashley, K. (1951). The problem of serial order in behavior. In L. A. Jeffress (Ed.), Cerebral mechanisms in behavior: The Hixon Symposium (pp. 112-136). New York: Wiley.

Marslen-Wilson, W. D. (1987). Functional parallelism in spoken wordrecognition. Cognition, 25, 71-102.

McKelvie, S. J. (1987). Learning and awareness in the Hebb Digits Task. The Journal of General Psychology, 114, 75-88.

Page, M. P. A., \& Norris, D. (2009). A model linking immediate serial recall, the Hebb repetition effect and the learning of phonological word forms. Philosophical Transactions of the Royal Society of London. Series B, Biological Sciences, 364(1536), 3737-3753.

Parmentier, F. B., Andrés, P., Elford, G., \& Jones, D. M. (2006). Organization of visuo-spatial serial memory: Interaction of temporal order with spatial and temporal grouping. Psychological Research, $70,200-217$.

Pelli, D. G. (1997). The VideoToolbox software for visual psychophysics: Transforming numbers into movies. Spatial Vision, 10, 437-442.

Ryan, J. (1969). Grouping and short-term memory: Different means and pattern of grouping. Quarterly Journal of Experimental Psychology, 21, 137-147.

Shallice, T., \& Warrington, E. K. (1970). Independent functioning of verbal memory stores: A neuropsychological study. Quarterly Journal of Experimental Psychology, 22, 261-273.

Smalle, E. H. M., Bogaerts, L., Simonis, M., Duyck, W., Page, M. P. A., Edwards, M. G., \& Szmalec, A. (2016). Can chunk size differences explain developmental changes in lexical learning? Frontiers in Psychology, 6:1925. https://doi.org/10.3389/fpsyg.2015.01925

Thorn, A. S. C., \& Page, M. P. A. (2009). Interactions between short-term and long-term memory in the verbal domain. Hove: Psychology Press.

Publisher's note Springer Nature remains neutral with regard to jurisdictional claims in published maps and institutional affiliations. 